\title{
La reseña como puerta de entrada a los géneros académicos: un estudio de caso
}

\author{
Gabriel S. Valdés-León ${ }^{1 \star}$ y Luis G. Barrera² \\ (1) Universidad de Las Palmas de Gran Canaria, Escuela de Doctorado. Calle Juan de Quesada, 30, 35001 Las Palmas \\ de Gran Canaria, Las Palmas, España. (correo-e: gabo_valdes@hotmail.com) \\ (2) Universidad Católica Silva Henríquez, Grupo de investigación Prácticas lectoras y escritura académica, Escuela de \\ investigación y Posgrado, Gral. Jofré 462, Santiago, Chile (correo-e: Ibarrera@ucsh.cl)
}

* Autor a quien debe ser dirigida la correspondencia

Recibido Nov. 25, 2019; Aceptado Ene. 21, 2020; Versión final Mar. 9, 2020, Publicado Ago. 2020

\begin{abstract}
Resumen
El objetivo de este estudio es indagar en el potencial que posee el género discursivo reseña, como género de formación, en el proceso de transición de estudiantes de pedagogía de primer año hacia un dominio discursivo propio del ámbito académico en el que se insertan. Se analizaron las reseñas académicas producidas por 36 estudiantes de primer año de la carrera de pedagogía en castellano de una universidad chilena, católica y privada. El instrumento de evaluación es una rúbrica con tres subcompetencias: lingüística, discursiva y sociolingüística. Los resultados muestran que la implementación de una unidad didáctica con énfasis en la producción de reseñas académicas desde una perspectiva sociocognitiva y pragmalingüística permitió que los estudiantes mejoraran la calidad de sus escritos en la competencia discursiva y sociolingüística. Se concluye que los resultados marcan una tendencia que abre un campo de estudios en torno al valor de la reseña en la educación terciaria.
\end{abstract}

Palabras clave: alfabetización académica; géneros académicos; reseña académica; didáctica de la lengua

\section{The review as a gateway to academic genres: a case study}

\begin{abstract}
The objective of this study is to investigate the potential of the discursive gender review, as a training genre, in the transition process of first year pedagogy students towards a discursive domain of the academic field in which they are inserted. This study analyzes the results of the academic reviews produced by 36 first-year undergraduate students of the Spanish pedagogy at a Chilean, catholic, and private university. A rubric was used as an evaluation instrument that considered three sub-competencies: linguistic, discursive, and sociolinguistic. The results show that the implementation of a didactic unit with emphasis on the production of academic reviews from a sociocognitive and pragmalinguistic perspective allowed students to improve the quality of their writings, especially in aspects related to discursive and sociolinguistic competence. In conclusion, the results show a tendency that opens a field of study on the value of reviews in tertiary education.
\end{abstract}

Keywords: academic literacy; academic genres; academic review; language teaching 


\section{INTRODUCCIÓN}

Uno de los grandes desafíos que actualmente enfrentan las universidades se relaciona con la inserción efectiva de los estudiantes a la comunidad discursiva propia de su disciplina. Esto ha motivado que las universidades se hagan cargo del problema a través de diversos mecanismos: writing centers, cursos de escritura o mediante la integración de actividades de lectoescritura académica como parte del currículum. Un ejemplo de ello es el esfuerzo que las instituciones estadounidenses, desde hace ya varias décadas, han realizado para fortalecer la escritura en la educación superior: "...en primer año suele abordarse como un curso obligatorio al comienzo del estudio universitario en los Estados Unidos. Dependiendo de la política de la universidad, los estudiantes de primer año pueden tomar cursos de escritura correctiva/básica, composición de primer año o cursos de escritura más avanzados [la traducción es nuestra]" (Curry y Lillis, 2003). No obstante, en cualquiera de estos escenarios, el papel del docente es fundamental (Zabalza, 2009; Ponce, Gómez y Viteri, 2019). Por ello, es necesario que quienes guían procesos de enseñanza-aprendizaje asuman que su rol formador se resume en la capacidad para alfabetizar a sus estudiantes en las prácticas de lectura y escritura propias de su disciplina.

En el ámbito hispánico, las iniciativas se han llevado de diferentes maneras atendiendo a la particularidad de cada contexto. A modo de ejemplo, podemos señalar las experiencias colombianas en la estrategia de tutorías, entre ellas, el trabajo sobre percepciones realizado por Chois-Lenis et al. (2017), que da cuenta, a través de un estudio de caso, de los beneficios que reportan tanto tutor como tutorado; por su parte, CalleArango (2020) realizó una revisión en los espacios existentes en las universidades colombianas y encuentra 24 iniciativas que, en el marco de la alfabetización académica, mantienen la utilización de tutorías.

Si se observa el caso de Chile, prácticamente en todas las universidades existen cursos transversales que, con diferentes nombres y perspectivas, apuntan al desarrollo de habilidades comunicativas. Asimismo, aún en el plano nacional, se han implementado algunos centros de escritura académica (en la Universidad Católica, la Universidad Alberto Hurtado y la Universidad de Chile, entre otras) los que, con diferentes alcances, se han preocupado por apoyar a los estudiantes de educación superior en este proceso de integración a una nueva comunidad discursiva.

En este contexto, el objetivo de este estudio de caso fue indagar en el potencial que posee el género discursivo reseña en el proceso de transición de estudiantes de pedagogía de primer año hacia un dominio discursivo propio del ámbito académico en el que se insertan. Para el diseño didáctico, se adoptó una perspectiva sociocognitiva y pragmalingüística (Didactex, 2003), complementada con los aportes de Navarro (2019) en los que profundizaremos en el siguiente apartado, y se elaboró a partir de la redacción de 5 reseñas críticas que abordaron textos sobre lingüística, educación y didáctica de la lengua; además, el módulo incluyó actividades de autoevaluación y evaluación entre pares. La muestra incluyó a la totalidad de la cohorte 2018 de Pedagogía en Castellano de la UCSH, vale decir, 36 estudiantes. La metodología del trabajo que aquí presentamos es un estudio de caso, de carácter preexperimental y enmarcada en los estudios sobre alfabetización académica.

Desde una perspectiva sencilla y directa, alfabetizar, según el DLE, significa "enseñar a leer y escribir". Carlino (2013), por su parte, señala que: “...alfabetizar académicamente equivale a ayudar a participar en prácticas discursivas contextualizadas..." (p. 370). Se alfabetiza a los integrantes de una comunidad educativa mediante la puesta en práctica de las normas, estrategias, procedimientos, formas y condiciones contextuales en que se produce o se procesa un texto de formato académico cuya circulación está destinada a un medio de su misma naturaleza. La meta última de este proceso es estimular el aprendizaje de quien ingresa a una institución universitaria y se orienta a "participar en la cultura discursiva de las disciplinas..." (Carlino, 2003).

Naturalmente, el proceso de alfabetización académica es complejo: implica abordar los procesos de lectura y escritura propios del nivel terciario de manera progresiva, guiando a los aprendientes desde los géneros más simples a los más complejos a través de recorridos pedagógicos amparados en decisiones institucionales que permitan al estudiante integrarse, poco a poco, en la comunidad discursiva propia de su disciplina (Lea y Street, 2006; Carlino, 2013; Arancibia, 2014; Baker, Bangeni, Burke y Hunma, 2019; Durmuşoğlu, Yüksel, Öztürk y Tömen, 2019).

Se trata entonces de enseñar el dominio de los diferentes géneros discursivos inherentes a las disciplinas y el currículo de una carrera universitaria de pregrado o posgrado (Sánchez Upegui, 2016; Navarro, 2018). Esto conlleva un proceso mediante el cual las instituciones en las que circulan textos de las ciencias y disciplinas especializadas propician no solo la enseñanza, las implicancias teóricas y las características estructurales y pragmáticas de determinados formatos discursivos, sino también los mecanismos inherentes a su producción oral o escrita, la difusión, el análisis y el procesamiento lector. El fenómeno ha sido 
estudiado y puesto en práctica en cuanto a su utilización e importancia en los diversos niveles de educación superior, desde el pregrado universitario hasta el doctorado (Carlino, 2003, 2013; Bolívar y Beque, 2011; Navarro y Abramovich, 2012; Mostacero, 2013; Regueiro Rodríguez y Sáez Rivera, 2013; Parodi y Burdiles, 2015; Sánchez Upegui, 2016). Adicionalmente, su esencia trae consigo la premisa según la cual el ingreso a una institución universitaria no supone en absoluto que haya concluido para el estudiante el aprendizaje de las técnicas de la lectura, la escritura y la producción y procesamiento de textos.

Lo expresado anteriormente compromete a la universidad y la fuerza a continuar contribuyendo con desarrollo de un proceso (meta) cognitivo que es mucho más complejo de lo que pudo creerse en el pasado. Esto significa ampliar el rango de lo que realmente significa alfabetizar: emprender un conjunto de acciones relacionadas con el aprendizaje de conceptos, estrategias, discurso, tecnolecto, formatos y otros asuntos propios de las disciplinas. Se relaciona con "la enseñanza de competencias situadas, significativas y transferibles a contextos nuevos y flexibles" (Navarro, 2018): enseñar a aprender y a transformar el conocimiento a través de la escritura, considerando como parte de esos nuevos contextos la democratización y diversidad de la educación del siglo XXI (Sobrero, Lara-Quinteros, Méndez y Suazo, 2018; Zavala, 2019). Lo anterior implica entender que un escritor competente es capaz de "comprender, aprender, negociar y adecuarse a las sofisticadas expectativas comunicativas de entornos distintos (Bazerman, 2013, citado en Navarro, Ávila y Gómez, 2019). Debe dejarse de lado el ritual didáctico que no pocas veces conduce a la creencia (a veces inconsciente, es verdad) de que el estudiante debe ejercitar el dominio de la escritura solo para responder evaluaciones (Carlino, 2003). El aprendiente tiene que internalizar que la escritura es para comunicarse con otros y que esos otros pueden ser sus pares.

No obstante, no debe confundirse la alfabetización académica con propuestas remediales dirigidas a mejorar la escritura en general y el procesamiento de textos (Carlino, 2013). El sentido de alfabetizar académicamente es diverso; está vinculado con cada una de las disciplinas relacionadas con un sistema curricular determinado. Su foco principal no es solamente el alumno; también implica al docente, porque se trata de mecanismo dialógico para la "construcción del conocimiento" (Bolívar y Beque, 2011), a partir de la apropiación y el dominio de un tipo específico de discurso, propio del área disciplinar que se practique y de la comunidad científico-discursiva en la que se desenvuelve la persona.

Resulta evidente, entonces, la importancia de acompañar los primeros años de universidad de los estudiantes (cfr. Carlino, 2003). A modo de ejemplo, podemos citar el trabajo de Roldán y Zabaleta (2016) en el que los autores investigan sobre las autopercepciones acerca de la escritura de alumnos de nuevo ingreso y confirman que este grupo encuentra mayores dificultades al momento de escribir que aquellos que se encuentran en cursos más avanzados. En esta misma línea, Ayala (2019) plantea las debilidades que los estudiantes universitarios de primer año que participaron de su estudio demuestran en el plano de la lectoescritura, lo que implica que "no pueden desarrollar argumentos válidos para un debate, análisis o discusión de un tema académico o científico." (p.2). Estos ejemplos relevan la necesidad de fortalecer la escritura en la universidad y, sobre todo, en los primeros años de estudio.

\section{LA RESEÑA Y SUS CARACTERÍSTICAS}

Este trabajo se limita a un género específico relacionada con una actividad curricular de la carrera de Pedagogía en Castellano de una universidad chilena, la reseña crítica, una mínima parcela de todo lo que tiene que ver con las disciplinas inherentes a una carrera de esta naturaleza, vinculada fundamentalmente con la enseñanza, pero también con el lenguaje propio de los distintos campos del saber implícitos en la lingüística y la literatura.

Más allá de las subcategorías que puedan existir, podemos delimitar dos tipos genéricos de reseñas: a) reseña resumen, cuya secuencia dominante suele ser expositivo-explicativa, con función descriptiva; b) reseña crítica, marcada textualmente por la argumentación y con una focalización en lo opinático, en la confrontación de puntos de vista, que pueden ser diversos, pero con dos infaltables, el del autor o autora original reseñado(a) y el del autor o autora de la reseña. Uno de sus rasgos primordiales es la exigencia de un alto nivel de "condensación conceptual" (Mostacero, 2013, p. 177). La principal motivación para elaborarla proviene del análisis de otro texto (el reseñado). Producirla acarrea algunas estrategias escriturales ineludibles: resumen, parafraseo, comparación e interpretación (con base fundamental en la inferencia), entre otras. Precisa, además, de conocimiento previo del tema, dominio del tecnolecto disciplinar, claridad para argumentar y, consecuentemente, para la emisión de juicios de valor y confrontación de opiniones (base evaluativa, Navarro y Abramovich, 2012). Por supuesto, se da por sentado que el reseñador debe haber atravesado por un proceso de entrenamiento inherente a los aspectos formales y comunicacionales de este género discursivo. Pragmáticamente, obliga al autor o autora a enfocar su texto con base en la prosa de lector (la que considera primordialmente a la audiencia, que en la realidad es de pares académicos). Estratégicamente, implica no solo inferir, sintetizar y mediar acerca de lo reseñado, sino también concretarse mediante un proceso de aplicación de macrorreglas que reduzca la temática, separe lo accesorio y focalice lo fundamental del objeto. 
En síntesis, la reseña crítica académica es un género cuya secuencia dominante debe ser la argumentación; es producida por un integrante de una comunidad académica, con el propósito de hacer conocer a los destinatarios (sus pares) puntos de vista acerca del objeto reseñado. La estructura retórica más sencilla es aquella que la divide en introducción, desarrollo descriptivo-argumentativo y conclusión(es). Junto con otras como el artículo, el ensayo, la monografía, el resumen, el examen, etc., se trata además de un género muy importante dentro de lo que se conoce como procesos de alfabetización académica, a los cuales nos hemos referido antes. Para este caso particular, el tipo de reseña analizado entra en la categoría de lo que Navarro y Abramovich (2012) catalogan como propia de las carreras universitarias. Sus rasgos diferenciales son de carácter pragmático: también debe tener un enfoque crítico, pero no está realmente dirigida a los pares de una comunidad académica específica por tratarse de un "género de formación" (Navarro, 2018, p. 26): usualmente es utilizada por el docente en la sala de clases para verificar comprobaciones y/o controles de lectura.

En términos generales, Navarro y Abramovich (2012) proponen una estructura en tres partes: contextualización, descripción y evaluación y conclusión. La primera de estas responde a las preguntas cuál es el tema, quién es su autor, cuándo y dónde se publicó el texto y qué importancia tiene; la segunda, se hace cargo de los temas que se abordan en el texto fuente, las perspectivas que esto sigue, las hipótesis que plantea y las fortalezas y debilidades que es posible evidenciar; finalmente, la conclusión se hace cargo de la visión general que el autor de la reseña posee sobre el texto fuente y, además, de la recomendación final. Mostacero, por su parte, señala que las reseñas poseen una estructura retórica sencilla, compuesta por encabezamiento, cuerpo y nombre del reseñador, de las cuales, la segunda corresponde a la más compleja, pues "Aquí se debe atender a la natural ordenación de partes o capítulos que tiene el texto fuente y así ofrecer una versión sugestiva" (Mostacero, 2013). Asimismo, el autor señala que la reseña crítica incorpora una doble visión -la del autor reseñado y la del reseñador-, visión que se debe desarrollar a lo largo de todo el documento y no solo al final del texto.

Para nuestros fines, optamos por entregar a la reseña crítica una estructura retórica más cercana a la propuesta por Navarro y Abramovich (2012), pero con algunos matices. Así, considerando que este trabajo representa para la mayoría de los estudiantes del curso el primer acercamiento a un texto académico, abrimos la posibilidad de separar la descripción de la evaluación, pues se evidenciaron dificultades al momento de articular las voces reseñador-reseñado de manera interrelacionada.

Desde el punto de vista didáctico, hemos abordado el género reseña a partir de los aportes de Didactext (2003) y Navarro (2019), quien adopta una perspectiva "significativa, articulada, socioconstructivista, crítica y explícita de la enseñanza y aprendizaje de los géneros discursivos". (p.23-24). En nuestra propuesta, se asumen estos criterios de la siguiente forma: "significativa" en tanto se abordaron textos disciplinares y se discutieron en clases; "articulada", pues los textos elaborados tenían como destinatarios tanto al docente como a sus pares; "socioconstructivista", ya que se deconstruyó la estructura retórica de una reseña académica, lo que les permitió comprender los aspectos modulares del género; "crítica"; tanto respecto de la construcción del género como en el plano del contenido del texto reseñado; y explícita, debido a que la planificación de sus escritos consideraba la reflexión en torno al objetivo del trabajo y las expectativas de los eventuales lectores.

Finalmente, dado el potencial pedagógico que ofrece la reseña, seleccionamos este género para favorecer la inserción de los estudiantes de primer año de Pedagogía en Castellano en la comunidad discursiva de la que aspiran formar parte. Para ello, implementamos una secuencia didáctica que, desde una perspectiva sociocognitiva y pragmalingüística, permitió que la calidad de sus trabajos mejorara, sobre todo, en aquellos aspectos relacionados con las convenciones propias de la disciplina, aspectos considerados en la competencia lingüística y discursiva.

\section{METODOLOGÍA}

En este apartado, se presenta un desglose de aquellos elementos que configuraron la metodología de la investigación. Asimismo, se incluye una síntesis de la unidad didáctica (tabla 1) que entrega mayores precisiones acerca de las decisiones pedagógicas que se adoptaron.

\section{Diseño}

La investigación fue diseñada como un estudio de caso, de carácter pre-experimental, longitudinal y con medición pre y postest.

\section{Participantes}

En el estudio participó la totalidad de la cohorte 2018, vale decir, 36 estudiantes pertenecientes a la carrera de Pedagogía en Castellano de una universidad chilena, católica y privada. El curso en el que se enmarcaron las actividades fue Producción oral y escrita, el que tiene una doble importancia en el perfil de 
egreso, pues contribuye con el desarrollo de las competencias escriturales tanto en el plano disciplinar como transversal. Cabe señalar que el itinerario académico de los estudiantes no considera instancias previas en las que se aborde la escritura con este doble enfoque.

\section{Módulo didáctico}

Para la elaboración del módulo didáctico, hemos trabajado con el curso de Producción oral y escrita, de carácter obligatorio, el que se imparte en dos clases semanales de dos horas pedagógicas cada una. Si bien la escritura es una habilidad transversal, el curso posee un marcado carácter disciplinar en tanto las manifestaciones textuales representan un medio de comunicación y, a la vez, un objeto de estudio para los profesores de lengua en formación. Además, tomando en consideración la importancia de integrar progresivamente a los estudiantes de nuevo ingreso a la comunidad discursiva de la que aspiran formar parte, se optó por guiar la producción de una reseña crítica, género discursivo que, dada la simpleza de su estructura retórica, resulta adecuado para alumnos de primer año, poco o nada alfabetizados para la educación superior. Sobre esa base, se planificó una secuencia didáctica que se organizó tal como lo muestra la tabla 1:

Tabla 1: síntesis de la organización de la unidad didáctica

\begin{tabular}{|l|l|l|}
\hline \multicolumn{3}{|l|}{$\begin{array}{l}\text { Objetivo del módulo: Redactar reseñas críticas relacionadas con temas disciplinares, respetando las convenciones } \\
\text { propias de la comunidad discursiva en la cual los estudiantes comienzan a integrarse. }\end{array}$} \\
\hline Producto & $\begin{array}{l}\text { Cantidad } \\
\text { de clases }\end{array}$ & Descripción \\
\hline $\begin{array}{l}\text { Reseña 1 } \\
\text { (Pretest) }\end{array}$ & 1 & $\begin{array}{l}\text { Se solicita a los estudiantes la elaboración de una reseña crítica del texto La escritura } \\
\text { como proceso: cuatro dificultades de la enseñanza universitaria (Carlino, 2004). Para } \\
\text { entregar el documento, contaron con una semana de plazo. }\end{array}$ \\
\hline Reseña 2 & 4 & $\begin{array}{l}\text { Se solicita a los estudiantes la elaboración de una reseña crítica del texto decálogo } \\
\text { didáctico de la enseñanza de la composición (Cassany, 2001). El trabajo se guio clase a } \\
\text { clase respetando tres momentos: planificación (una clase), textualización (dos clases), } \\
\text { revisión (una clase). }\end{array}$ \\
\hline Reseña 3 & 4 & $\begin{array}{l}\text { Se solicita a los estudiantes la elaboración de una reseña crítica de un texto académico a } \\
\text { elección sobre escritura en la universidad. El trabajo se guio clase a clase respetando tres } \\
\text { momentos: planificación (una clase), textualización (dos clases), revisión (una clase). }\end{array}$ \\
\hline Reseña 4 & 4 & $\begin{array}{l}\text { Se solicita a los estudiantes la elaboración de una reseña crítica del texto La escritura } \\
\text { como proceso (Cuervo y Flórez, 1992). El trabajo se guio clase a clase respetando tres } \\
\text { momentos: planificación (una clase), textualización (dos clases), revisión (una clase). }\end{array}$ \\
\hline $\begin{array}{l}\text { Reseña 5 } \\
\text { (Postest) }\end{array}$ & 1 & $\begin{array}{l}\text { Se solicita a los estudiantes la elaboración de una reseña crítica del texto Fortalecimiento } \\
\text { de la competencia comunicativa escrita en estudiantes de primer año de pedagogía en } \\
\text { castellano a través de una estrategia tutorial entre pares (Valdés, Mendoza y Galaz, } \\
\text { 2017). Para entregar el documento, contaron con una semana de plazo. }\end{array}$ \\
\hline
\end{tabular}

Cabe destacar que se utilizaron variadas estrategias didácticas: para la etapa de planificación, mapas mentales, mapas conceptuales, lluvia de ideas y esquemas; para la etapa de textualización, preguntas orientadoras sobre el contenido, la situación y el texto; y para la etapa de revisión, coevaluación, autoevaluación y evaluación formativa por parte del docente. En síntesis, el módulo didáctico se diseñó desde una perspectiva sociocognitiva y pragmalingüística (Didactext, 2003) y asegurando el carácter "significativo, articulado, socioconstructivista, crítico y explícito de la enseñanza y aprendizaje de los géneros discursivos" (Navarro, 2019). Se elaboró a partir de la redacción de 5 reseñas críticas que abordaron textos sobre lingüística, educación y didáctica de la lengua. El primero de estos escritos fue utilizado como pretest, mientras que el último, como postest. La escritura se trabajó en parejas y se guio clase a clase de manera procesual, con énfasis en el carácter social de la escritura.

\section{Instrumento}

La recolección de información se realizó a través de una rúbrica, que es el resultado de la adaptación del instrumento que propuso el Instituto Vasco de Educación en el año 2013 y que fue validada mediante juicio experto por tres profesionales del ámbito de la educación, número que considera su experticia en el área y la diversidad del conocimiento (Escobar-Pérez y Cuervo-Martínez, 2008, citados en Robles y Rojas, 2015). El instrumento valora tres dimensiones, a saber, competencia sociolingüística (objetivo, registro y cohesión), competencia discursiva (cohesión, estructura y coherencia) y competencia lingüística (ortografía puntual, acentual y literal). Para cada criterio se asignó 1, 3 o 5 puntos de acuerdo con el nivel de logro (no logrado, medianamente logrado, logrado). Este instrumento ha sido utilizado, con algunas adaptaciones, en trabajos anteriores orientados también a la evaluación de la escritura en educación superior (cfr. Valdés, Ceballos, 
Valdebenito, Farías, 2019). Considerando que esta experiencia forma parte de las actividades formativas del curso Producción oral y escrita, el instrumento se circunscribe tan solo a los tres aspectos señalados pues son aquellos que, de acuerdo con el avance del programa de estudios, resulta necesario evaluar. Pese a esto, somos conscientes de la necesidad de implementar, progresivamente, instrumentos de evaluación de la escritura de carácter holístico, que la aborden atendiendo a su complejidad (Navarro, Ávila y Gómez, 2019).

\section{Procedimiento}

Se aplicó la rúbrica para evaluar cada una de las reseñas producidas, tanto al inicio y al final del módulo (pre y postest) como durante el desarrollo de este.

\section{Proceso de análisis de datos}

La rúbrica se aplicó al momento de evaluar cada uno de los trabajos generados en las cinco instancias que consideró el módulo didáctico. Además, con el fin de aumentar la precisión de la evaluación, se recurrió a tres métodos: proceso sistemático de evaluación, entrenamiento de los correctores y correcciones matemáticas para resolver discrepancias. Respecto de este último aspecto, se llevó a cabo a través del promedio de los puntajes obtenidos por los dos evaluadores a cargo de esta etapa.

\section{RESULTADOS}

En este apartado, presentaremos los resultados del trabajo organizados de la siguiente manera: primero, la relación entre los puntajes del pre y postest para cada una de las competencias (Figura 1); luego, el resultado obtenido, de manera global, por cada uno de los equipos que conformaron la muestra del estudio. En la figura 1 se presenta una comparación entre los resultados obtenidos en el pretest y en el postest, los que corresponden a la reseña 1 y 5 , respectivamente. Cabe señalar que se evidencia un incremento proporcional en cada una de las competencias que el instrumento de evaluación consideró, no obstante, los aspectos relacionados con el léxico, la gramática y la ortografía -a saber, competencia lingüística- fue el aspecto que tuvo un menor aumento. Por su parte, elementos como el objetivo y el registro utilizado, evaluados en la competencia sociolingüística, presentaron el mayor incremento. Finalmente, la competencia lingüística presenta también una mejoría, sin embargo, sigue siendo el aspecto que evidencia mayor debilidad.

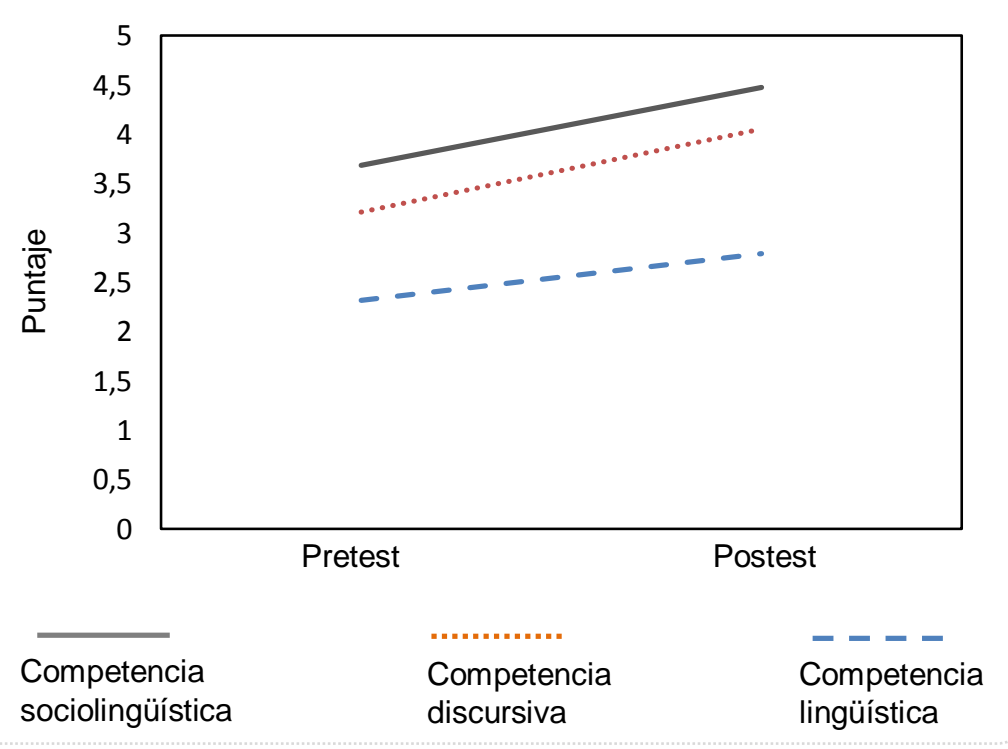

Fig. 1: Resultados del pre y postest

Como se puede observar en la figura 2, la reseña 5 (postest) presenta, en términos generales, un mejor desempeño que la reseña 1 (pretest). Los casos en los que los estudiantes no lograron incrementar el puntaje de su evaluación representan a menos de un tercio de la muestra (grupo 2, 4, 10, 11 y 13), en tanto que solo dos equipos bajaron su puntaje. Evidentemente, la tendencia es una mejora en la mayoría de los trabajos, mucho mayor en los estudiantes que obtuvieron un bajo puntaje en la evaluación inicial (p.e., el grupo 7). 


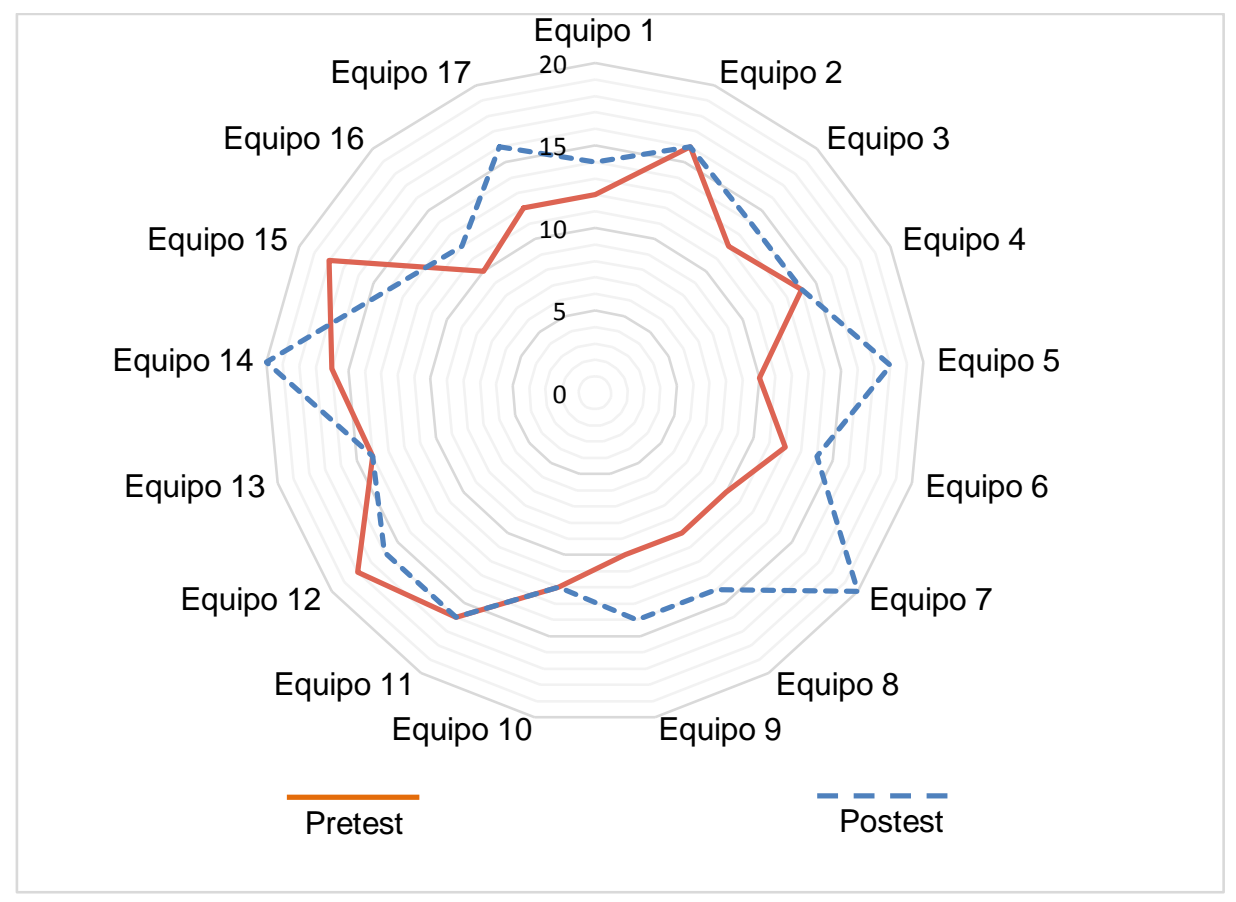

Fig. 2: Resultados globales por equipo

\section{DISCUSIÓN}

Uno de los resultados más interesantes de este estudio tiene que ver con el incremento en la competencia discursiva, pues se relaciona estrechamente con una de las principales demandas de la educación superior: integrar progresivamente a los aprendientes a las comunidades discursivas propias de su disciplina (Geisler, 2013). En este sentido, el trabajo coincide con los resultados que entregan investigaciones que, a través de ejercicios sistemáticos de escritura situada y contextualizada, evidencian mejoras en aspectos relacionados con la organización de la información, con el objetivo del escrito y con la adecuación a las convenciones propias de la esfera discursiva (Usman, 2015; Hódar, Pérez-Martínez et al., 2016; Abegglen, Burns et al., 2016; Malouff, Rooke et al. 2018).

Por su parte, el hecho de que la competencia lingüística haya obtenido los resultados más bajos no resultó ser una sorpresa, pues en esta dimensión se consideró la ortografía acentual, literal y puntual. Al respecto, trabajos como los de Cáceres-Lorenzo (2014), Tankó y Csizér (2018), Rashidi y Mazdayasna (2016), entre otros muchos, dan cuenta de las falencias que los estudiantes universitarios, sobre todo los de nuevo ingreso, presentan en este ámbito. De todas maneras, es posible explicar también estos resultados debido a que el foco del trabajo de los estudiantes, dada la perspectiva didáctica basada en géneros que hemos adoptado, se centra en dimensiones de mayor demanda cognitiva, por lo que podrían verse descuidados elementos microlingüísticos como los señalados.

No obstante lo anterior, más allá de adoptar una postura punitiva y desligarse del problema, los docentes deben asumir que su trabajo consiste en integrar progresivamente a los futuros profesionales a la comunidad discursiva de la que aspiran formar parte. En otras palabras, implica asumir que "La habilidad de leer y evaluar un texto de un escritor novato se halla en «hacerse el ciego" ante los problemas ortográficos -al menos en este primer borrador-y concentrarse primero en qué quiere decir el estudiante (...) Es la maravilla de contemplar la evolución de una ensayista en construcción" (Sánchez, 2007).

Ahora bien, no podemos atribuir las mejoras en los resultados relacionados con la competencia escrita solo a las actividades relacionadas con este estudio de caso, pues es indudable que el trabajo en otras asignaturas impacta también en su formación como escritores. Lo que sí podemos establecer con mayor propiedad es que la reseña, como género de formación, tuvo un impacto positivo en el proceso de transición hacia la cultura académica universitaria, sobre todo, a través actividades de lectoescritura de textos disciplinares.

Por último, si bien la mayor parte de los estudiantes presentó una mejora en la calidad de sus textos, hay algunos trabajos -tan solo dos- que disminuyeron el puntaje obtenido. Este dato, que desde el punto de vista estadístico es comprensible y aceptable, pedagógicamente es un llamado de atención: invita a reflexionar en torno a los factores que incidieron en que un grupo de estudiantes no haya alcanzado el nivel de desarrollo esperado y, consecuentemente, remirar, desde las propias prácticas docentes hasta los 
elementos externos (sobrecarga académica, problemas socioafectivos...), qué podría haber incidido en este resultado. Evidentemente, indagar en esos motivos queda fuera del alcance de este trabajo.

\section{CONCLUSIONES}

Los resultados generales del trabajo indican que una secuencia didáctica que trabajó la producción del género discursivo reseña, desde una perspectiva sociocognitiva y pragmalingüística de la escritura, demostró ser útil para contribuir con el desarrollo de la competencia discursiva, sociolingüística y lingüística, lo que impactó positivamente el proceso de alfabetización académica de los aprendientes. Si bien los resultados no son generalizables, debido a lo acotado de la secuencia, marcan una tendencia que abre un interesante campo de estudios en torno al valor de la reseña en la educación terciaria.

\section{REFERENCIAS}

Abegglen, S., Burns, T., y Sinfield, S., Utilizing Critical Writing Exercises to Foster Critical Thinking in Diverse First-Year Undergraduate Students and Prepare Them for Life Outside University, 4, 266-277. Double Helix (2016)

Adam, J.M., Les Textes: Types et Prototypes. Paris, Nathan. (1992)

Ayala, C., Competencia comunicativa: la argumentación en estudiantes del primer ciclo de la UCSG: un estudio de casos. Revista Tecnológica Ciencia y Educación Edwards Deming 3(2), 41-49. (2019)

Arancibia Aguilera MC., The design of a rubric to evaluate laboratory reports in astronomy: academic literacy in the disciplines. Profile Issues in TeachersProfessional Development., 16(1), 153-65 (2014)

Bajtín, M. M., Aesthetic of the verbal creation. México DF, México: Siglo XXI. (1982)

Baker S, Bangeni B, Burke R, Hunma A., The invisibility of academic reading as social practice and its implications for equity in higher education: a scoping study. Higher Education Research \& Development, 2;38(1), 142-56. (2019)

Bolívar, A. y Beque, R., Lectura y escritura para la investigación. Universidad Central de Venezuela-Consejo de Desarrollo Científico y Humanístico, Caracas, Venezuela (2011)

Cáceres-Lorenzo, M. T., Variables en el desarrollo de la reflexión metalingüística (L1) de alumnos plurilingües en el primer año universitario. Revista Española de Lingüística Aplicada, 27(2), 275-296. (2014)

Calle-Arango, L., Centros y programas de escritura en las IES colombianas. doi: 10.11144/Javeriana.m12-25.cpei, magis, Revista Internacional de Investigación en Educación, 12 (25), 77-92. (2020).

Carlino, P., Alfabetización académica: un cambio necesario, algunas alternativas posibles. Educere, 6 (20), 409-420. (2003)

Carlino, P., Alfabetización académica 10 años después. Revista Mexicana de Investigación Educativa, 18, (57), 355-381, (2013)

Cassany, D., Decálogo didáctico de la enseñanza de la composición. Glosas didácticas: revista electrónica internacional de didáctica de las lenguas y sus culturas. (acceso: 16 Octubre 2019)

Ciapuscio, G., Tipos textuales. Buenos Aires, Argentina: Ciclo básico común, (1994)

Chois-Lenis, P., Casas-Bustillo, A., López-Higuera A., Prado-Mosquera, D., Cajas-Paz, E. Percepciones sobre la tutoría entre pares en escritura académica Magis. Revista Internacional de Investigación en Educación, 9(19), 165-184. (2017)

Curry, M., y Lillis, T., Issues in academic writing in higher education. Teaching academic writing: A toolkit for higher education, 1-18. (2003)

DIDACTEXT, Modelo sociocognitivo, pragmalingüístico y didáctico para la producción de textos escritos. Didáctica. Lengua y literatura, 15, 077-104. (2003)

Durmuşoğlu K., Yüksel, l., Öztürk, Y. y Tömen, M., Turkish Academics' Foreign Language Academic Literacy: A Needs Analysis Study. International Journal of Instruction, 12(1), 717-736. (2019)

Geisler, C., Academic literacy and the nature of expertise: Reading, writing, and knowing in academic philosophy. Routledge. (2013).

Hódar, J. A., Pérez-Martínez, C. y otros tres autores, Correct your own exam. Exercises for university students to develop writing skills in biology. In SHS Web of Conferences (Vol. 26, p. 01079). EDP Sciences. (2016)

Lea, M. R., y Street, B. V., The "academic literacies" model: Theory and applications. Theory into practice, 45(4), 368377. (2006)

Loureda Lamas, O., Introducción a la tipología textual. Arco/Libros, Madrid, España (2003)

Malouff, J., Rooke, S., y Schutte, N., Simple Strategies Academics Can Use to Help Students Improve Their Writing Skills. Online Submission. (2018)

Mostacero, R., Construcción de la reseña crítica mediante estrategias metacognitivas. Lenguaje, 41 (1), 169-200, (2013) 
Navarro, F., Más allá de la alfabetización académica: las funciones de la escritura en educación superior. En M. A. Alves \& V. Iensen Bortoluzzi (Eds.), Formação de Professores: Ensino, linguagens e tecnologías, 13-49. RS: Editora Fi, Porto Alegre, Brasil (2018)

Navarro, F. Aportes para una didáctica de la escritura académica basada en géneros discursivos, doi: https://dx.doi.org/10.1590/1678-460x2019350201_DELTA: Documentação de Estudos em Lingüística Teórica e Aplicada, 35(2), e2019350201. (2019)

Navarro, F. y Abramovich, A. L. La reseña académica. En L. natale (coord.), En carrera: la lectura y la escritura de textos académicos y profesionales, 39-60. Universidad Nacional de General Sarmiento, Buenos Aires, Argentina (2012)

Navarro, F. Ávila, N. y Gómez, G. Validez y Justicia: hacia una evaluación significativa en pruebas estandarizadas de escritura. Revista Meta: Avaliação 11.31, 1-35. (2019)

Parodi, G. y Burdiles, G., Leer y escribir en contextos académicos y profesionales: géneros, corpus y métodos. : Ariel, Santiago, Chile (2015)

Ponce Ruiz, D., Gómez, A. y Viteri, D. Modelo de formación multidimensional del Docente universitario en UNIANDES Quevedo con énfasis en la Investigación Científica. Dilemas Contemporáneos: Educación, Política y Valores ,6 (2019)

Rashidi, N., Mazdayasna, G. Impact of Genre-Based Instruction on Development of Students' Letter Writing Skills: The Case of Students of Textile Engineering, doi: 10.22055/rals.2016.12094, Research in Applied Linguistics, 7(2), 55-72. (2016)

Regueiro Rodríguez, M. L. y Sáez Rivera, D. M., El español académico. Guía práctica para la elaboración de textos académicos. Arco Libros, Madrid, España (2013)

Robles Garrote, P. y Rojas, M. D. C., La validación por juicio de expertos: dos investigaciones cualitativas en Lingüística aplicada. Revista Nebrija de Lingüística Aplicada, 18 (2015)

Roldán, L. y Zabaleta, V. Lectura y escritura. Autopercepción del desempeño en estudiantes universitarios. Revista Iberoamericana de Diagnóstico y Evaluación, 2(42), 27-38. (2016)

Sánchez, C. La escritura ortográfica en estudiantes universitarios. Textos de didáctica de la lengua y la literatura, (45), 99-106. (2007)

Sánchez Upegui, A. A., Alfabetización académica: leer y escribir desde las disciplinas y la investigación. Revista lasallista de investigación, 13 (2), 200-209, (2016)

Sobrero, V., Lara-Quinteros, R., Méndez, P., y Suazo, B. Equidad y diversidad en universidades selectivas: la experiencia de estudiantes con ingresos especiales en las carreras de la salud. Pensamiento Educativo. Revista de Investigación Educacional Latinoamericana, 51(2), 152-164. (2014)

Tankó, G., y Csizér, K., Individual Differences and Micro-argumentative Writing Skills in EFL: An Exploratory Study at a Hungarian University. In University Writing in Central and Eastern Europe: Tradition, Transition, and Innovation, 149-166. Springer, Cham. (2018)

Usman, M., Teaching Model of Learning English Writing at University. Jurnal IImiah Peuradeun, 3(3), 441-450. (2015)

Valdés, G., Ceballos, F., Valdebenito, A. y Farías, M. Impacto de una experiencia interdisciplinaria para desarrollar la redacción de cuentos en el aula de Historia desde la metodología A+S, doi: https://doi.org/10.17162/au.v9i3.379, Apuntes universitarios, 9(3). (2019).

Zavala, V. Justicia sociolingüística para los tiempos de hoy. doi:10.17533/udea.ikala.v24n02a09 Íkala, revista de lenguaje y cultura, 24(2), 343-359. (2019)

Zabalza. M. Ser profesor universitario hoy. En Revista La Cuestión Universitaria, 5, 69-81. (2009) 
